\title{
Does the presence of a trainee compromise success of biliary cannulation at ERCP?
}

\section{다(1) $(9)$}

\author{
Authors \\ John Warwick Frost, Arun Kurup, Sharan Shetty, Neil Fisher
}

Institution

Dudley Group of Hospitals NHS Foundation Trust, Gastroenterology, Dudley, UK nad Northern Ireland

submitted 6.11.2016

accepted after revision 1.2 .2017

Bibliography

DOI https://doi.org/10.1055/s-0043-105579 |

Endoscopy International Open 2017; 05: E559-E562

(c) Georg Thieme Verlag KG Stuttgart · New York

ISSN 2364-3722

Corresponding author

John Warwick Frost, Dudley Group of Hospitals NHS

Foundation Trust - Gastroenterology, Russell's Hall

Hospital, Pensnett Road, Dudley DY1 2HQ, United Kingdom

of Great Britain and Northern Ireland

Phone: +07903290821

john.w.frost@outlook.com

john.frost@nhs.net

\section{ABSTRACT}

Background and study aims Findings in the literature are conflicting on whether trainee involvement in endoscopic retrograde cholangiopancreatography (ERCP) procedures is detrimental to cannulation success rates. We addressed this in a prospective study, where cannulation success with or without trainee presence was the primary outcome measure.
Patients and methods We prospectively recorded data on 2 senior endoscopists and their trainees over an 18month period for ERCPs in patients with a virgin ampulla. Presence or absence of a trainee at ERCP procedures was pragmatic, reflecting their other service or training commitments or annual leave. For trainee presence, the training protocol allowed them 6 minutes of supervised time in which to achieve biliary cannulation after reaching the ampulla. Study outcome measures included cannulation success, time to cannulation, technique, whether this was achieved independently by the trainee, and complications. Results There were 219 procedures recorded and analyzed (134 with a trainee, 85 without). Three trainees were involved. Selective biliary cannulation was achieved in 201 (92\%) of cases. When a trainee was present, cannulation was successful in $122 / 134$ procedures $(91 \%)$, compared to $79 / 85$ (93\%) with a senior endoscopist alone $(P=0.8$, Fisher's exact test). Mean time to biliary cannulation with a trainee present was 7 minutes, compared with 5 minutes with no trainee. Mean time for successful independent cannulation by the trainee was 4 minutes, and 9 minutes for a consultant following a trainee's attempt. There were no serious adverse events.

Conclusion Our study shows that with this training protocol, involvement of a trainee on a routine secondary care ERCP list does not impair biliary cannulation success, and does not prolong a subsequent attempt by the senior endoscopist if initially unsuccessful. These findings support the involvement of trainees in routine ERCP lists with this training protocol.

\section{Introduction}

ERCP has evolved from that of a diagnostic procedure in which its role has largely been replaced by noninvasive imaging, to an interventional procedure, whose role is as important as ever in the face of an aging population with its high incidence of pancreaticobiliary pathology. For the senior endoscopist there are at least 3 challenges that characterize each case: patient tolerance, procedural success, and post-procedure complications. Together with these challenges there is a need to train the next generation of endoscopists, and balancing these challen- ges with the quality standards expected of a modern ERCP service is difficult [1]. There is evidence from several case series that involvement of trainees may compromise cannulation success rates [2-5]. There is also possible concern that involvement of a trainee may make subsequent cannulation more difficult for the senior endoscopist, thereby increasing risk of complications such as pancreatitis [6,7]. These concerns may dissuade some practitioners from offering training other than to highly motivated and capable trainees [8]. However, in contrast, other case series have not found such a detrimental effect of trainee involvement in ERCP cases $[3,7,9,10]$. None of the 
published case series addressed trainee involvement and cannulation success rate as a primary outcome measure, so we addressed this in a prospective case series in a secondary care setting in the UK. The aim of the study was to address whether involvement of a trainee in ERCP cases with a virgin ampulla has a detrimental effect on biliary cannulation success rates, compared with a senior endoscopist doing the procedure alone.

\section{Patients and methods}

The setting was a secondary care ERCP service in an acute district general hospital with an annual caseload of approximately 330 ERCP cases per year. The service is led by 2 senior endoscopists, each with over 5 years' experience in providing ERCP training to specialist registrars within the deanery. Specialist registrars with an interest in ERCP are given basic training in ERCP subject to completion of accreditation in upper gastrointestinal endoscopy. Procedures are done with conscious sedation. The trainee is allowed to start the procedure and once the ampulla has been reached (with or without assistance) and a stable position achieved, the trainee is allowed 6 minutes within which to achieve cannulation of the selected duct under supervision. If cannulation is achieved the trainee continues with the procedure if the trainer feels it appropriate to do so. If not then the trainer takes over. Cases are routinely done with a shortwire system (Cook UK), using a wire-led or cannula-led approach to the ampulla according to Ampullary morphology and the trainer's judgement. Secondary techniques are used at the discretion of the trainer. Successful cannulation is defined as deep cannulation of the desired duct with the guidewire and cannula.

For this study, procedural details were recorded on a proforma ( $\triangleright$ Fig. 1). Presence or absence of a trainee was not randomized but was pragmatic; the Trust has 2 specialist registrars (usually allocated because of an interest in learning ERCP) but trainee presence on ERCP lists is inconsistent because of other priorities (acute general medical rota, mandatory regional training days, annual and study leave etc). Therefore the study reflected normal working practice. The primary outcome measure was success of biliary cannulation; secondary outcomes included time to cannulation, technique of cannulation, whether independent cannulation achieved by the trainee, and whether there were any immediate significant complications.

\section{Results}

Over an 18-month period from September 2013 to March 2015 we collected data from 219 ERCP procedures with a virgin ampulla. Among these cases a trainee was present for 134 (61\%) and absent for 85 (39\%). Procedural indications reflected the normal working practice of a district general hospital; pre-procedure imaging suggested gallstone disease in $67 \%$, biliary stricture in $7 \%$ and uncertain or other pathology in $26 \%$. Procedures requiring selective cannulation of the pancreatic duct were excluded from the study. Predicted grade of difficulty varied with 133 patients classified as grade 1 (61\%); 81 patients as grade $2(37 \%)$; and 3 patients as grade $3(2 \%)$ using the Cot-

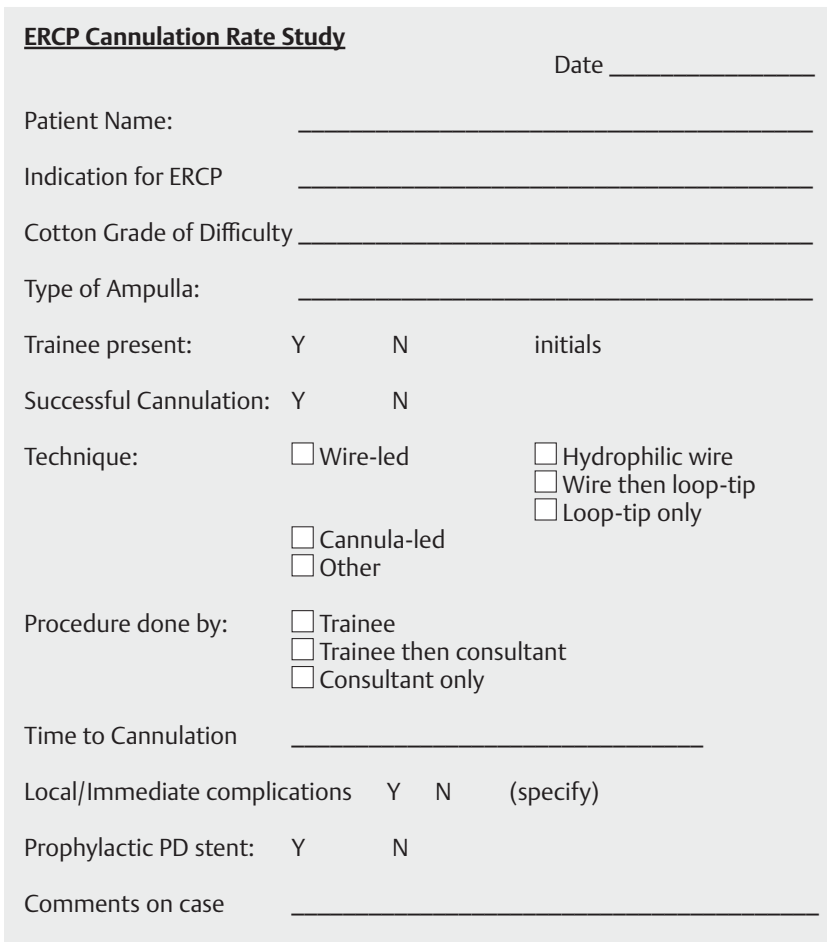

Fig. 1 Study proforma.

ton scale [11]. Three trainees were included in the data capture, all of whom had performed $<50$ ERCP procedures each prior to starting training at this center. For a summary of results please refer to $\triangleright$ Table 1 .

\section{Primary outcome}

\section{Cannulation rates}

Selective cannulation was achieved in 201 (92\%) of the 219 procedures. When a trainee was present, cannulation was successful in 122/134 procedures (91\%); when there was no trainee present, cannulation was successful in 79/85 (93\%) $(P=$ 0.8 , Fisher's exact test).

For the 2 individual consultants, successful cannulation rates with a trainee were $92 \%$ and $90 \%$ respectively, and without a trainee were $93 \%$ and $93 \%$ respectively ( $P=1$ for both, Fisher's exact test).

\section{Secondary outcomes}

Time to cannulation

Mean time to biliary cannulation with a trainee present was 7 minutes, compared with 5 minutes with no trainee $(P<0.01$, Student's t-test). Mean time for successful independent cannulation by the trainee was 4 minutes, and 9 minutes for a consultant following a trainee's attempt. Therefore when a consultant took over from a trainee after the allotted 6 minutes, a further 3 minutes were required on average for successful cannulation, i.e. a shorter cannulation time compared with a consultant-only procedure. 
Table 1 Summary of results.

\begin{tabular}{|c|c|c|c|c|}
\hline Number of procedures & \multicolumn{4}{|l|}{219} \\
\hline Trainee present & \multicolumn{4}{|l|}{$61 \%$} \\
\hline \multirow[t]{3}{*}{ Cotton grade of difficulty } & 1 & \multicolumn{3}{|l|}{$61 \%$} \\
\hline & 2 & \multicolumn{3}{|l|}{$37 \%$} \\
\hline & 3 & \multicolumn{3}{|l|}{$2 \%$} \\
\hline \multicolumn{5}{|l|}{ Primary outcomes } \\
\hline \multirow[t]{3}{*}{ Successful biliary cannulation } & Overall & \multicolumn{3}{|l|}{$92 \%$} \\
\hline & Trainee present & \multicolumn{2}{|l|}{$91 \%$} & $P=0.8$ \\
\hline & Trainee absent & \multicolumn{3}{|l|}{$93 \%$} \\
\hline \multicolumn{5}{|l|}{ Secondary Outcomes } \\
\hline \multirow[t]{4}{*}{ Mean time to cannulation } & \multirow[t]{3}{*}{ Trainee Present } & Independent & \multicolumn{2}{|l|}{$4 \mathrm{~min}$} \\
\hline & & Trainee then consultant & \multicolumn{2}{|l|}{$9 \mathrm{~min}$} \\
\hline & & Overall & \multicolumn{2}{|l|}{$7 \mathrm{~min}$} \\
\hline & \multicolumn{2}{|l|}{ Trainee Absent } & \multicolumn{2}{|l|}{$5 \mathrm{~min}$} \\
\hline \multirow[t]{7}{*}{ Techniques } & \multirow[t]{3}{*}{ Wire-led } & Trainee & \multicolumn{2}{|l|}{$46 \%$} \\
\hline & & Consultant & \multicolumn{2}{|l|}{$61 \%$} \\
\hline & & Overall & \multicolumn{2}{|l|}{$54 \%$} \\
\hline & \multirow[t]{3}{*}{ Cannula-led } & Trainee & \multicolumn{2}{|l|}{$50 \%$} \\
\hline & & Consultant & \multicolumn{2}{|l|}{$36 \%$} \\
\hline & & Overall & \multicolumn{2}{|l|}{$41 \%$} \\
\hline & \multicolumn{2}{|l|}{ Unspecified (overall) } & \multicolumn{2}{|l|}{$5 \%$} \\
\hline \multirow[t]{5}{*}{ Complications } & \multicolumn{2}{|c|}{ Minor ampullary bleeding } & \multicolumn{2}{|l|}{2 cases } \\
\hline & \multicolumn{2}{|c|}{ False guidewire passage } & 2 cases & \\
\hline & Prophylactic stents & Overall & 21 cases & \\
\hline & & Trainee present & 12 cases & $P=0.8$ \\
\hline & & Trainee absent & 9 cases & \\
\hline
\end{tabular}

\section{Cannulation technique}

Fifty-four percent of successful procedures were wire-led and $41 \%$ were cannula-led (5\% unspecified). For trainee success, $46 \%$ of successful procedures were wire-led and $50 \%$ were cannula-led (4\% unspecified). Secondary techniques to achieve CBD cannulation were used in 14/219 (6\%) of the cases, and were successful in $11 / 14$ ( $80 \%)$. The secondary techniques used were locked pancreatic duct wire cannulation (10 cases), pre-cut sphincterotomy ( 3 cases), and needle-knife fistulotomy ( 2 cases). In the 10 cases where a locked pancreatic duct wire cannulation was required, $8(80 \%)$ were with a trainee present, and $2(20 \%)$ without. Prophylactic pancreatic duct stents were placed in $21 / 219$ cases $(10 \%) ; 12$ in cases with the trainee present, 9 in cases without $(P=0.8)$.

\section{Trainee success rate}

Overall trainee success rate for independent cannulation was $34 \%$ with no major immediate complications recorded. For the
3 individual trainees, successful cannulation rates were $46 \%$, $43 \%$, and $11 \%$.

\section{Complications}

There were no significant immediate complications. Ampullary bleeding and false guidewire passage were recorded in 2 cases each; none required any further intervention after the procedure. This study was not designed to monitor prospectively for later complications but within the study period there were no cases of complicated pancreatitis (i.e. requiring further intervention); no cases of procedure-related perforation, and no procedure-attributed deaths.

\section{Discussion}

Our study shows that having a trainee present on a routine secondary care ERCP list does not impair biliary cannulation. Overall, successful cannulation was achieved in $91 \%$ of cases with a 
trainee present in this series. This exceeds the threshold of $85 \%$ set out in the 2014 BSG standards framework for ERCP and earlier published case series from the UK $[1,12]$. High success rates were observed for both trainers working with trainees. In addition (within the constraints of this study) a failed attempt by the trainee on average did not then prolong the consultant's time on the ampulla, despite all trainees being junior ( $<50$ procedures prior to this placement and independent trainee cannulation success rate of only $34 \%$ within this study period). While having a trainee present may prolong the total length of time it takes to achieve biliary cannulation, this did not appear to lead to increased rates of immediate post-procedural complications or need for prophylactic pancreatic duct stenting. Although we found a slightly higher likelihood of needing a secondary cannulation technique with a trainee present, the number of cases was still relatively small and accounted for only $5 \%$ of procedures.

Our data are encouraging for trainees but other published case series remain conflicting. The reasons for conflicting outcomes from other case series may reflect the retrospective nature of many series and a lack of standardisation for trainee involvement. Variables that may impact on success or failure of cannulation where a trainee is involved may include the use of propofol or other general anaesthetic agents, caseload and case mix (including whether the ampulla is "virgin" or not), use of short-wire vs. long-wire techniques, the skills and prior experience of both the trainee and trainer, and the time allowed for the trainee $[13,14]$. Most of these variables remain unexplored but they may help explain differences in trainee-related outcomes in other case series. A further prospective study, with a larger cohort of patients, trainees and consultants might help to clarify the variation observed amongst such studies.

Some case series have suggested a threshold of around 200 ERCPs for competence to perform ERCPs independently, and the low trainee success rate for independent cannulation in our study (with trainees being at an early stage of training) accords with this $[13,15,16]$. This low success rate is commensurate with a previous audit from the UK [2]. The success rate may have been improved by allowing the trainee more time at the ampulla; one recent prospective study identified an allowance of 10 minutes as the optimum trainee time [14]. Our protocol of allowing 6 minutes is not evidence-based but has evolved as a pragmatic approach allowing for other factors including patient tolerance, ampullary "compliance", hyoscine half-life, and list caseload. A longer trainee time, while desirable for trainee outcomes, may depend on provision of deeper levels of anaesthesia and support being available.

\section{Conclusion}

Our pragmatic ERCP training protocol is likely to be reflective of other ERCP training in the UK $[2,17]$. There may be more effective ways to deliver training in terms of achieving better trainee success rates, but we hope that our findings will reassure experienced endoscopy trainers that ERCP training can be delivered via the protocol we have described without compromising their own cannulation success rates.

\section{Competing interests}

None

References

[1] Wilkinson M et al. ERCP - The Way Forward: A standards Framework. BSG 2014

[2] Williams E], Taylor S, Lombard M et al. Are we meeting the standards set for endoscopy? Results of a large-scale prospective survey of endoscopic retrograde cholangio-pancreatograph practice. Gut 2007; 56: $821-829$

[3] Williams E], Ogollah R, Lombard M et al. What predicts failed cannulation and therapy at ERCP? Results of a large-scale multicenter analysis. Endoscopy 2012; 44: 674-683

[4] Peng C, Nietert PJ, Romagnuolo J et al. Predicting native papilla biliary cannulation success using a multinational Endoscopic Retrograde Cholangiopancreatography (ERCP) Quality Network. BMC Gastroenterol 2013; 13: 147

[5] Shekhar C, Shetty S, Fisher NC. PTU-002 Ercp Cannulation; Evaluation of a Wire-led technique for biliary access in a training centre. Gut 2014; 63: A37-A38

[6] Wicks ACB, Robertson GSM, Veitch PS. Structured training and assessment in ERCP has become essential for the Calman era. Gut 1999; 45: $154-156$

[7] Freeman ML, DiSario JA, Roel JP et al. Risk factors for post-ERCP pancreatitis: a prospective, multicenter study. Gastrointest Endosc 2001; 54: $425-434$

[8] Baillie J, Testoni PA. Are we meeting the standards set for ERCP? Gut 2007; 56: $744-746$

[9] DeBenedet AT, Elmunzer B], Schoenfeld PS et al. Intraprocedural quality in endoscopic retrograde cholangiopancreatography: a metaanalysis. Am J Gastroenterol 2013; 108: 1696 - 1704

[10] Ekkelenkamp VE, De Man R, Koch A et al. Prospective evaluation of ERCP performance: Results of a nationwide quality registry. Endoscopy 2015; 47: $503-507$

[11] Cotton PB, Eisen G, Romagnuolo J et al. Grading the complexity of endoscopic procedures: results of an ASGE working party. Gastrointest Endosc 2011; 73: 868-874

[12] Sheppard DP, Craddock S], Wilkinson ML et al. ERCP cannulation success benchmarking: Implications for certification and validation. Frontline Gastroenterol DOI: 10.1136/flgastro-2014-100473

[13] Voiosu T, Bengus A, Mateescu B et al. Trainee caseload correlates with ERCP success rates but not with procedure-related complications: results from a prospective study (the QUASIE cohort). Endosc Int Open 2016; 04: E409-E414

[14] Pan Y, Zhao L, Guo X et al. Appropriate time for selective biliary cannulation by trainees during ERCP - a randomized trial. Endoscopy 2015; 47: 688-695

[15] Jowell PS, Baillie J, Phillips Bute B et al. Quantitative Assessment of Procedural Competence: A Prospective Study of Training in Endoscopic Retrograde Cholangiopancreatography. Ann Intern Med 1996; 125: $983-989$

[16] Verma D, Gostout C], Adler DG et al. Establishing a true assessment of endoscopic competence in ERCP during training and beyond: a singleoperator learning curve for deep biliary cannulation in patients with native papillary anatomy. Gastrointestinal Endoscopy 2007; 65: 394 400

[17] Ramakrishnan S, Crosbie J, Oppong K et al. One year prospective audit of ERCP across one Hospital Trust. Gut 2005; 54 (Suppl. 02): a1 - a117 